\title{
Author Index for Volume 70
}

Published online: 27 February 2018

(c) Japan Antibiotics Research Association 2018

\begin{tabular}{|c|c|c|c|c|c|}
\hline Abdelfattah, MS & 601 & Bang, WY & 737 & Chen, H-P & 1104 \\
\hline Abe, $\mathrm{T}$ & 246,435 & Bao, L & 915 & Chen, J-C & 801,1009 \\
\hline Aboshi, T & 1133 & Barbeira, PJS & 277 & Chen, L & 1104 \\
\hline Adachi, $\mathrm{H}$ & 1146 & Batta, G & 152,664 & Chen, $\mathrm{M}$ & 158 \\
\hline Adelin, E & 1043 & Bekker, OB & 871 & Chen, R & 970 \\
\hline Afiyatullov, SS & 856 & Beppu, T & 361 & Chen, W & 962 \\
\hline Agasar, D & 1000 & Bereczki, I & 664 & Chen, X & 795, 991 \\
\hline Aguilar-Colomer, & 259 & Berg, DE & 339 & Chen, Z & 632,923 \\
\hline Ahamed, AAP & 754 & Bernasconi, A & 73 & Cheng, J & 147 \\
\hline Ahn, JS & 102,323 & $\mathrm{Bi}, \mathrm{X}$ & 991 & Cheramie, MN & 65 \\
\hline Ajito, $\mathrm{K}$ & $52,655,888,1112$ & Bigi, R & 962 & Cherian, PT & 65 \\
\hline Akbarsha, MA & 754 & Bischoff, KM & 136,1065 & Cheung, A & 1009 \\
\hline Alharbi, NS & 754 & Biswas, K & 747 & Chiba, J & 179 \\
\hline Alharbi, SA & 754 & Blackburn, JA & 1122 & Chiba, $S$ & 967 \\
\hline Ali, A & 212 & Blaskovich, MAT & 3 & Chmielewski, M & 781 \\
\hline Amemiya, M & 791 & Blumenthal, A & 1097 & Choi, W-S & 1065 \\
\hline Amin, $\mathrm{M}$ & 763 & Bonfill, M & 1043 & Chou, WKW & 625 \\
\hline Ando, $\mathrm{K}$ & 995 & Bonnet, R & 809 & Choudhury, JD & 747 \\
\hline Ando, $\mathrm{O}$ & 79,84 & Borbás, A & 152,664 & Cisalpino, PS & 277 \\
\hline Ando, $\mathrm{T}$ & 443 & Borelli, BM & 277 & Clemente, L & 809 \\
\hline Andreu, D & 231 & Brown, $\mathrm{P}$ & 386 & Coates, ARM & 1020 \\
\hline Angius, F & 962 & Brown, $\mathrm{T}$ & 98 & Coelho, PMZ & 680 \\
\hline Anwar, C & 1133 & Bruhn, DF & 65 & Cooper, MA & 3 \\
\hline Aoki, S & 1070 & Brunati, C & 73 & Cossé, AA & 136 \\
\hline Arahata, S & 443 & Bryant, B & 130 & Courvalin, $\mathrm{P}$ & 400 \\
\hline Araújo, N & 680 & Bull, AT & 448 & Crespo, E & 828 \\
\hline Ariefta, NR & 1129,1133 & Burgess, DS & 974 & Crombie, A & 1097 \\
\hline Arima, S & 647 & Burgess, KMN & 721 & Crump, A & 495 \\
\hline Arora, $\mathrm{P}$ & 212 & Butler, MS & 3 & Cruz, JCS & 73 \\
\hline Arunachalam, C & 754 & Bykov, EE & 871 & Csávás, M & 152,664 \\
\hline Asada, Y & 695,979 & & & Cui, Z & 819 \\
\hline Asadullah, 115 & & Campion, JJ & 974 & Culp, E & 366 \\
\hline Asai, A & 443 & Cane, DE & 625 & & \\
\hline Asami, Y & 395,921 & Caniça, M & 809 & Dai, M & 1138 \\
\hline Asenjo, JA & 448 & Capon, RJ & 1097 & Dairi, $\mathrm{T}$ & 798 \\
\hline Avula, SR & 954 & Cavalcante, CSP & 231 & Daletos, G & 726 \\
\hline \multirow[t]{2}{*}{ Aziz, M } & 115 & Cen, S & 105 & Danilenko, VN & 871 \\
\hline & & Chang, Z & 832 & Dastager, SG & 1000 \\
\hline Baba, H & 1070 & Che, Q & 174 & Davis, RW & 859 \\
\hline Babar, MM & 308 & Chen, A-I & $190,219,297$ & Dawson, MJ & 386 \\
\hline Baltz, RH & 639 & Chen, G-Y & 313,823 & de Almeida, KC & 122 \\
\hline Bang, S & 737 & Chen, $\mathrm{H}$ & 915,1138 & de Faria Junior, $\mathrm{C}$ & 122 \\
\hline
\end{tabular}




\begin{tabular}{|c|c|c|c|c|c|}
\hline de Matos Cardoso Per & digão, G & Fukuda, T & 96,590 & $\mathrm{He}, \mathrm{N}$ & 970 \\
\hline de Oliveira Motta, D & 122 & Fukumoto, A & 562,568 & $\mathrm{He}, \mathrm{Q}$ & 970 \\
\hline de Sá, NP & 277 & Furman, B & 781 & Hemphill, CFP & 726 \\
\hline Demain, AL & $347,520,828$ & Furukawa, S & 41,45 & Herczegh, P & 152,664 \\
\hline Deng, J-y & 285 & Furuuchi, T & 246 & Hijikawa, Y & 304 \\
\hline Deng, X & 1029 & Futamura, Y & $102,222,705$ & Hillenmeyer, ME & 859 \\
\hline Deng, XZ & 853 & & & Hiltunen, $\mathrm{T}$ & 805 \\
\hline Deng, Z & 90 & Gao, M-y & 216 & Hirai, Y & 52,1112 \\
\hline Deshpande, A & 65 & Gaspari, E & 73 & Hiramatsu, K & 142,264 \\
\hline Dezhenkova, LG & 871 & $\mathrm{Ge}, \mathrm{HM}$ & 853 & Hirano, S & 929 \\
\hline Didar, A & 845 & Gelber, M & 828 & Hirose, $\mathrm{T}$ & $568,574,878$ \\
\hline Ding, G & 801,1138 & Goff, GL & 1043 & Hirotani, M & 695,979 \\
\hline Ding, Z-T & 313,743 & Goodfellow, M & 448 & Hirota-Takahata, Y & $79,84,179,981$ \\
\hline Dittmer, DP & 962 & Goto, $\mathrm{K}$ & 272 & Hong, B & 158 \\
\hline Doadrio, JC & 259 & Gould, CA & 625 & Hong, W & 832 \\
\hline Donadio, $\mathrm{S}$ & 73 & Graça, R & 809 & Hong, Y-S & 323 \\
\hline Dong, J & 788 & Grammatikova, & 871 & Hori, R & 675 \\
\hline $\mathrm{Du}, \mathrm{M}-\mathrm{n}$ & 219 & Grillot-Courvali & $\mathrm{n}, \mathrm{C}$ & Horikoshi, R & 272 \\
\hline Duan, Y & 414,819 & $\mathrm{Gu}, \mathrm{J}$ & 285 & Horimatsu, Y & 680 \\
\hline Duan, Y-R & 200,823 & $\mathrm{Gu}, \mathrm{K}-\mathrm{b}$ & 733 & Hotta, K & 335 \\
\hline \multirow[t]{2}{*}{ Dyshlovoy, SA } & 856 & $\mathrm{Gu}, \mathrm{Q}-\mathrm{G}$ & 174 & Houssen, WE & 448 \\
\hline & & $\mathrm{Gu}, \mathrm{Y}-\mathrm{C}$ & 801 & $\mathrm{Hu}, \mathrm{M}$ & 313 \\
\hline Ebel, R & 448 & Guda, MR & 1065 & $\mathrm{Hu}, \mathrm{X}$ & 158 \\
\hline Eguchi, T & 423 & Gugala, N & 775 & $\mathrm{Hu}, \mathrm{Z}$ & 1029 \\
\hline Eguchi, Y & 251 & Gullo, V & 828 & Huang, G-L & 823 \\
\hline El-Elimat, $\mathrm{T}$ & 553 & Guo, Q-G & 147 & Huang, $\mathrm{H}$ & 819,1029 \\
\hline Elofsson, $\mathrm{M}$ & 937 & Guo, W & 174,970 & Huang, J & 190 \\
\hline Espíndola, L & 277 & Guo, Z & 414 & Huang, $\mathrm{P}$ & 715 \\
\hline \multirow[t]{2}{*}{ Esteban, J } & 259 & Gupta, VK & 212 & Huang, T & 90 \\
\hline & & Gurjarpadhye, A & 308 & Huang, X & 991 \\
\hline Falcão, CB & 231 & & & Huang, Y & 200,414 \\
\hline Falk, SP & 918 & Hägglund, U & 937 & Hubbard, ATM & 1020 \\
\hline Falzone, M & 828 & Hamada, M & 995 & Hurdle, JG & 65 \\
\hline Fang, S-T & 1047 & Hamamoto, H & $41,204,685$ & Hwang, BY & 323 \\
\hline Fang, X & 105 & Han, L & 991 & & \\
\hline Feng, $\mathrm{T}$ & 1104 & Hana, S & 708 & Ibrahim, A & 721 \\
\hline Feng, TJ & 301 & Hanaki, H & $562,568,574,878$ & Ibrahim, D & 222 \\
\hline Feola, DJ & 974 & Hanatani, N & 647 & Ichimaru, $\mathrm{N}$ & 395 \\
\hline Fernandes, $\mathrm{P}$ & 527 & Harada, R & 798 & Ida, $\mathrm{T}$ & 246 \\
\hline Fernández-Lobato, M & 404 & Hartman, TM & 1065 & Idris, $\mathrm{H}$ & 448 \\
\hline Ferreira, E & 809 & Harunari, E & 590 & Igarashi, $\mathrm{M}$ & $251,582,611,699$ \\
\hline Filho, CRS & 122 & Harvey, CJB & 859 & & 1078,1150 \\
\hline Fischer, CR & 859 & Harvey, RD & 1020 & Igarashi, Y & $595,607,708$ \\
\hline Fontenelle, ROS & 231 & Hasegawa, T & 179 & Iida, $\mathrm{K}$ & 675 \\
\hline Forrest, A & 98 & Hashimoto, M & 45 & Iijima, $\mathrm{K}$ & 1078,1150 \\
\hline Franco, OL & 122 & Hashimoto, T & 616 & Iijima, M & 791,1146 \\
\hline Froyman, R & 878 & Hashimoto, Y & 435 & Ikeda, A & 798 \\
\hline Fujie, A & 41,45 & Hashizume, H & 699 & Ikeda, $\mathrm{H}$ & 109,616 \\
\hline Fujikura, K & 911 & Hatano, M & $582,611,1078,1150$ & Ikegaya, A & 443 \\
\hline Fujimaki, T & 328,595 & Hayakawa, M & 995 & Ikoma, K & 995 \\
\hline Fujiwara, T & 582 & Hayakawa,Y & 196 & Imada, C & 590 \\
\hline Fukamizo, T & 251 & Hayashi, C & $582,1078,1150$ & Imaizumi, $\mathrm{T}$ & 675 \\
\hline Fukuda, D & 79,84 & $\mathrm{He}, \mathrm{K}-\mathrm{Y}$ & 823 & Imoto, $\mathrm{M}$ & $109,328,595$ \\
\hline
\end{tabular}


Imran, YMM

Inahashi, Y

Inamura, $S$

Inaoka, DK

Inayathullah, M

Ingianni, A

Ino, C

Inukai, Y

Ishibashi, M

Ishii, M

Ishikawa, N

Ishimoto, Y

Isogai, E

Isogai, Y

Ito, $\mathrm{M}$

Itoh, M

Ivanets, EV

Iwadate, Y

Iwanami, F

Iwatsuki, M

Izawa, M

Izumikawa, M

Jackson, MA

Jalasvuori, M

James, KD

Jang, J-H

Jang, J-P

Jaspars, M

Ji, N-Y

Jia, A

Jia, H-M

Jia, W-Q

Jiang, B

Jiang, $\mathrm{T}$

Jiang, Y

Jiang, Z

Jiao, RH

Jiménez, A

Johann, S

Jones, $\mathrm{K}$

Josten, M

$\mathrm{Ju}, \mathrm{J}$

Jun-Li, W

Kakeya, H

Kalinovsky, AI

Kamisuki, S

Kanasaki, R

Kanda, Y

Kanno, S-i

Kao, D

\begin{tabular}{|c|c|c|c|c|}
\hline 754 & Kassack, MU & 726 & Koomsiri, W & 1142 \\
\hline 1142 & Kataoka, T & 929 & Korn, VL & 828 \\
\hline 246 & Kato, C & 611 & Korolev, AM & 871 \\
\hline 304 & Kato, N & 632 & Koseki, T & 1129,1133 \\
\hline 308 & Kato, $\mathrm{T}$ & 718 & Kozuma, S & 79,84 \\
\hline 962 & Katz, L & 378 & Kreiswirth, BN & 671 \\
\hline 695,979 & Katz, N & 680 & Krishnan, BR & 130 \\
\hline 251 & Kaur, S & 832 & Kromann, S & 944 \\
\hline 601 & Kawada, M & $1078,1146,1150$ & Kubota, Y & $791,1078,1150$ \\
\hline 771,907 & Kawahara, T & 226 & Kudo, F & 423 \\
\hline 601 & Kawamori, F & 443 & Kulengowski, B & 974 \\
\hline 179,981 & Kawasaki, T & 196 & Kumano, T & 435 \\
\hline 142 & Kawashima, S & 1070 & Kumura, K & $52,655,888,1112$ \\
\hline 708 & Kaweewan, I & 208 & Kumura, M & 655 \\
\hline 718 & Keasling, JD & 378 & Kunisada, T & 791 \\
\hline 226 & Kelly, R & 1087 & Kuraya, N & 79,84 \\
\hline 856 & Khalil, ZG & 1097 & Kurepina, N & 671 \\
\hline 179,981 & Khan, F & 115 & Kurosawa, E & 179,981 \\
\hline 1078,1150 & Khan, G & 828 & Kushida, N & 987 \\
\hline $395,562,568$, & Khan, MN & 115 & Kuzuyama, T & 616,811 \\
\hline $574,911,921$ & Khan, SI & 115 & Kwon, B-M & 1065 \\
\hline 196 & Khosla, C & 859 & Kyoso, T & 607 \\
\hline 226 & Khudyakova, YV & 856 & & \\
\hline & Kido, Y & 304 & Lacey, E & 1097 \\
\hline 1122 & Kigawa, T & 251 & Lacriola, CJ & 918 \\
\hline 805 & Kim, C-J & 323 & Lara, MB & 122 \\
\hline 130 & Kim, K-M & 308 & Laudeman, CP & 130 \\
\hline 102,323 & Kim, S & 737 & Leathers, TD & 136 \\
\hline 102 & Kim, SU & 1057 & Lee, $\mathrm{C}$ & 737 \\
\hline 448 & Kim, TG & 1057 & Lee, H-J & 737 \\
\hline 1047 & Kim, Y-P & 562,568 & Lee, JK & 323 \\
\hline 1138 & Kim, Y & 607 & Lee, RE & 65 \\
\hline 801 & Kimata, S & 196 & Lee, S-H & 1065 \\
\hline 1026 & Kimura, K-i & 429, 685, 929, & Lee, TH & 1057 \\
\hline 158 & & 1129,1133 & Lei, H & 991 \\
\hline 801 & Kimura, T & 921,1142 & Lei, X & 158 \\
\hline $200,414,991$ & Kita, K & 304,647 & Leidy, C & 238 \\
\hline 158 & Kitani, S & 1004 & Lemire, JA & 775 \\
\hline 853 & Kizuka, M & 179 & Lenhard, JR & 98 \\
\hline 404 & Kleefeld, G & 878 & $\mathrm{Li}, \mathrm{D}$ & 174 \\
\hline 277 & Kobayashi, H & 179,981 & $\mathrm{Li}, \mathrm{E}$ & 743,923 \\
\hline 828 & Kobayashi, K & 96,435 & $\mathrm{Li}, \mathrm{F}$ & 174,193 \\
\hline 1009 & Kobayashi, M & 435 & $\mathrm{Li}, \mathrm{H}-\mathrm{Q}$ & 147 \\
\hline 819 & Kobayashi, Y & $695,921,979$ & Li, H-r & 166 \\
\hline \multirow[t]{2}{*}{1000} & Kodani, S & 208 & $\mathrm{Li}, \mathrm{J}-\mathrm{b}$ & $297,832,1065,1122$ \\
\hline & Koebberling, J & 878 & Li, J-M & 187 \\
\hline 718 & Kohda, Y & 1146 & $\mathrm{Li}, \mathrm{J}-\mathrm{s}$ & 216 \\
\hline 856 & Kojima, Y & 574 & $\mathrm{Li}, \mathrm{J}-\mathrm{L}$ & 788 \\
\hline 691 & Komal, R & 828 & $\mathrm{Li}, \mathrm{K}$ & 285 \\
\hline 41 & Komatsu, H & 1070 & $\mathrm{Li}, \mathrm{L}$ & 944,1138 \\
\hline 691 & Komatsu, M & 616 & $\mathrm{Li}, \mathrm{M}$ & 987 \\
\hline 331 & Kondo, K & 798 & $\mathrm{Li}, \mathrm{P}$ & 193 \\
\hline 553 & Kondoh, Y & 429 & Li, W & 737 \\
\hline
\end{tabular}




\begin{tabular}{|c|c|c|c|c|c|}
\hline Li, W-J & $47,320,795,853$ & Matsumoto, Y & 771,907 & Ngeow, YF & 832 \\
\hline Li, X-z & 313,715 & Matsuzaki, M & 304 & Nihira, T & 1004 \\
\hline $\mathrm{Li}, \mathrm{Y}-\mathrm{G}$ & 301,733 & Mattila, S & 805 & Nishimura, S & 718 \\
\hline $\mathrm{Li}, \mathrm{Z}$ & 819 & McAlpine, JB & 492,551 & Nishimura, Y & 699,1146 \\
\hline Li, Z-H & 1104 & McEwan, AR & 448 & Nishiyama, M & 616 \\
\hline Lim, CL & 222 & Mei, Q & 166 & Nitta, H & 1004 \\
\hline Lima, AO & 911 & Meinke, PT & 671 & Niu, S-B & 801 \\
\hline Lima, TB & 122 & Miao, F-P & 1047 & Niwa, M & 929 \\
\hline Lin, J & 743 & Miller, MJ & 292 & Nogawa, T & $102,222,705$ \\
\hline Lin, $S$ & 90 & Miller, PA & 292 & Noh, E-Y & 737 \\
\hline Lin, W & 726 & Minami, A & 632 & Nomoto, A & 582 \\
\hline Lin, $X$ & 788 & Mirani, Za & 115 & Nonaka, K & 395,911 \\
\hline Lin, Y & 987 & Mitomi, M & 272 & Nong, X-H & 763,1047 \\
\hline Linde-Arias, AR & 680 & Miura, $\mathrm{T}$ & 967 & Noorani, KPM & 754 \\
\hline Liras, $\mathrm{P}$ & 534 & Miyano, R & 921 & Norberg, HS & 937 \\
\hline Liu, B & 166 & Miyazaki, S & 995 & Nurjanto, HH & 1133 \\
\hline Liu, H & 105,915 & Miyoshi, H & 395 & & \\
\hline Liu, J-k & 1104 & Miyoshi, N & 142 & Oberlies, NH & 553 \\
\hline Liu, L & 743 & Mizumoto, K & 582 & O'Brien, RV & 859 \\
\hline Liu, M & 715 & Mizuno, F & 695,979 & Ochi, K & 25 \\
\hline Liu, X & 715,923 & Molloy, B & 404 & Ogasawara, Y & 798 \\
\hline Liu, Y & 788 & Momose, I & 542,791 & Ogita, N & 705 \\
\hline Lowry, B & 859 & Monteiro, JM & 1009 & Ogo, $\mathrm{N}$ & 443 \\
\hline $\mathrm{Lu}, \mathrm{C}$ & $320,795,1026$ & Morel, CM & 680 & Ohba, M & 443 \\
\hline $\mathrm{Lu}, \mathrm{S}$ & 718 & Morinaka, A & 246 & Ohnishi-Kameyam & na, $M$ \\
\hline Lu, X-R & 823 & Morita, K & 1070 & Ohshiro, $\mathrm{T}$ & 96 \\
\hline Ludwig, C & 878 & Moriya, K & 771,907 & Ohsumi, K & 45 \\
\hline \multirow[t]{2}{*}{ Lysenkova, LN } & 871 & Mossialos, E & 1087 & Ohtawa, M & 647 \\
\hline & & Mukherjee, J & 747 & Oikawa, H & 632 \\
\hline Ma, J & 991 & Murayama, T & 1133 & Oikawa, T & 607 \\
\hline Ma, K & 915 & Muroi, M & 429 & Ojala, V & 805 \\
\hline Ma, M & 200 & Muslihah, NI & 1133 & Oka, T & 443 \\
\hline Madeddu, C & 962 & & & Okada, M & 1146 \\
\hline Maffioli, SI & 73 & Naeimi, $\mathrm{H}$ & 845 & Okajima, $\mathrm{T}$ & 251 \\
\hline Magalhães, BS & 122 & Naesens, L & 152 & Okano, A & 222 \\
\hline Mahansaria, R & 747 & Nagai, K & 590 & Okawa, Y & 1129 \\
\hline Majewski, MW & 292 & Nagamitsu, T & 647 & Okazaki, M & 771,907 \\
\hline Malik, FA & 212 & Nagano, Y & 911 & Oku, N & 708 \\
\hline Manageiro, V & 809 & Nagayoshi, M & 1150,1078 & Okutomi, T & 888 \\
\hline Manaka, A & 264 & Nagumo, Y & 691 & Olsen, DB & 671 \\
\hline Manitchotpisit, P & 136 & Nair, DR & 1009 & Olsen, JE & 944 \\
\hline Manrique-Moreno, M & 238 & Nakagawa, H & 675 & Olsen, RH & 944 \\
\hline Manzano, M & 259 & Nakagawa, Y & 995 & Ombredane, AS & 122 \\
\hline Maria-Neto, S & 122 & Nakajima, M & 79,84 & Omelchuk, OA & 871 \\
\hline Martens, E & $347,520,527$ & Nakamura, I & 41,45 & Ōmura, $\mathrm{S}$ & $113,272,395$, \\
\hline Martín, JF & 534 & Nakashima, T & $395,921,1142$ & & $562,568,574,647$, \\
\hline Maruoka, T & 1070 & Namikoshi, M & 331,967 & & $680,695,878,911$, \\
\hline Maruyama, H & 878 & Nara, A & 616 & & $921,979,1142$ \\
\hline Masaki, S & 52 & Narusawa, S & 395 & Onaka, H & 865 \\
\hline Masaki, T & 45 & Naumann, TA & 1065 & Oñate-Garzón, J & 238 \\
\hline Matsui, $\mathrm{H}$ & $562,574,878$ & Nayda, V & 828 & O’Neill, AJ & 317 \\
\hline Matsumoto, A & $113,514,562$ & Naz, S & 115 & Onodera, $\mathrm{H}$ & 562,675 \\
\hline Matsumoto, M & 708 & Negishi, $\mathrm{S}$ & 705 & Onozawa, Y & \\
\hline
\end{tabular}


Ooi, N

Orfali, RS

Osada, H

Ostorházi, E

Otoguro, M

Ou, J

Ouazzani, J

Ouyang, Y

Owada, K

Oyama, K

Oyeleye, A

Paguigan, ND

Palnati, GR

Pan, A-j

Pan, G

Panbangred, W

Pang, X

Panthee, S

Parachin, NS

Parekh, MB

Park, D-J

Park, J-E

Pasupuleti, M

Patel, A

Patel, K

Patel, M

Paterson, IC

Patiño, E

Pearce, CJ

Pellizari, VH

Pereira, D

Pérez-Jorge, C

Perkins, C

Perret, F

Petersson, GA

Phay, N

Pieczykolan, M

Pinho, MG

Piras, E

Pizzolatti, MG

Polowy, K

Pompei, R

Pothineni, VR

Price, NPJ

Proksch, P

Qi, H

Qi, S-H

Qi, X

Qin, X-C

Quach, HT

Quadri, SR
317

726

102, 222, 429, 705

152, 664

995

320

1043

832

443

272

715

553

954

166

414

595

105

204

122

308

323

737

954

828

828

828

832

238

553

911

527

259

828

152

695, 921, 979

995

781

1009

962

277

130

962

308

136, 1065, 1122

726

187, 216, 219, 1026

763, 1047

970

788

929

1000
Rádis-Baptista, G

Raj, S

Raja, HA

Rajadas, J

Rasheed, MU

Rashid, Z

Rateb, ME

Ravin, A

Reehana, N

Reilly, I

Ren, B

Ren, H-J

Ren, J

Renwick, MJ

Retailleau, P

Rhöös, E

Ribeiro, SM

Riyaz-Ul-Hassan, S

Robbins, TF

Rodríguez-García, A

Rosa, CA

Rőth, E

Rotinsulu, $\mathrm{H}$

Ruan, B-H

Rudolf, JD

Ruotsalainen, $\mathrm{P}$

Ruwaili, JA

Sørensen, D

Sacchettini, JC

Saha, M

Sahl, H-G

Sahu, AK

Saif, LJ

Saito, R

Saito, S

Sakaguchi, SO

Sakai, K

Sakakibara, S

Sakamaki, Y

Sakamoto, H

Sakamoto, S

Sakata, N

Salam, N

Salim, AA

Sánchez, MB

Santhoshkumar, S

Sanz, E

Sasaki, T

Sashidhara, KV

Sassa, T

Sato, $\mathrm{T}$

Sato, Y
231

954

553

308

754

845

200, 448, 710

828

754

98

715

219, 970

923

1087

1043

937

122

212

859

534

277

152, 664

967

313

200,828

805

1000

721

1070

747

1009

1000

443

331

328, 595

911

911

246

246

1070

1146

226

147

1097

404

754

404

142

954

632

771, 907

52

Saugar, I

404

Saveljev, OY

871

Sawa, R

1078

Sawa, R

Scheffler, R

$582,595,611$,

$699,791,1146,1150$

Sekimizu, K 41, 204, 685, 771, 907

Serpe, R 962

Shchekotikhin, AE

Shen, B

871

Shen, Y

200, 414, 710, 828

Shi, J

795

Shibasaki, M

853

Shibusawa, S

791

878

Shibuya, Y

1078, 1150

Shikata, Y

109

Shim, SH

737

Shimizu, E

647

Shimizu, R

251,647

Shimizu, T

222

Shimura, S

691

Shinde, SS

632

Shinohara, Y 395

Shin-ya, K 226

Shinya, S

251

Shiomi, K

395, 457, 562, 568,

574, 647, 921, 1142

Shiono, Y

429, 929, 1129, 1133

Shiotsuki, T $\quad 395,695,921,979$

Si, S

158

Siddiqui, $\mathrm{S}$

828

Sidthipong, K

987

Silva, ON

122

Simpkin, VL

1087

Singh, LR

954

Singh, N

1033

Singh, SB

671

Smetanina, OF

856

Smith, S

130

Soga, S

675

Solow-Cordero, D 308

Son, H-U

1065

Son, SJ

1057

Soon, RL

98

Sosio, M 
Sumarah, MW

Sumida, N

Sumilat, DA

Sumilat, DA

Sun, L

Sun, M

Sunazuka, T 272, 568, 574, 680, 878

Sundin, C

Sureechatchaiyan, $\mathrm{P}$

Suzuki, A

Suzuki, K

Suzuki, M

Suzuki, S

Suzuki, T

Suzuki, Y

Svenningsen, SW

Szatmári, R

Szúcs, Z

Tabuchi, F

Taira, J

Takagi, $\mathrm{H}$

Takahashi, K

Takahashi, M

Takahashi, S

Takahashi, Y

Takase, S

Takata, $\mathrm{T}$

Takatsu, T

Takayama, Y

Také, A

Takehana, Y

Takishita, K

Tamura, T

Tan, RX

Tan, X

Tanabe, $\mathrm{K}$

Tanaka, I

Tanaka, M

Tang, J

Tang, Y

Tanigaki, R

Tansei, $\mathrm{K}$

Tao, $\mathrm{H}$

Tashiro, E

Tatsumi, R

Tatsuno, K

Tatsuta, K

Tavares, LC

Tawaraya, K

Tayebi, L

Tchoukoua, A
721

987

331

967

1138

320,795

937

726

96

680

562

304

1129, 1133

204

944

152, 664

152,664

771, 907

1070

443

911

590

102,222

113, 506, 514,

921,1142

45

246

84

246

113

611

911

995

853

832

264

981

179, 981

987

1104

929

708

788

109

304

771,907

$1,455,1083$

277

1133

308

1129
Thajuddin, $\mathrm{N}$

Thamchaipenet, A

Thao, NB

Tian, L

Tochio, N

Tokiwano, $\mathrm{T}$

Tokumitsu, $\mathrm{T}$

Tomioka, $\mathrm{T}$

Tomoda, H 96, 562, 590, 685, 1085

Torres, $\mathrm{R}$

Toyomasu, $\mathrm{T}$

Trier, $\mathrm{S}$

Tripathi, C

Tsuchida, $\mathrm{T}$

Tsuji, BT

Tsujii, N

Tsutsui, A

Tsutsumi, Y

Tsvetkov, VB

Tu, Z-C

Tuononen, $\mathrm{T}$

Turner, RJ

Uchida, $\mathrm{R}$

Uda, S

Ueda, K

Ueda, $\mathrm{S}$

$52,361,655,888,1112$

Ueki, M

Uesugi, S

Umei, $\mathrm{T}$

Umekita, M

Umemura, E

Umezawa, $\mathrm{K}$

Usui, T

Usukhbayar, $\mathrm{N}$

Utsumi, R

Uusitalo, $\mathrm{P}$

Vaidya, A

Vallet-Regí, M

Vanderlinden, E

Vandova, GA

Velpula, KK

Vermillion, KE

Vishwakarma, RA

von Amsberg, G

Vuong, D

Wada, S-i

Wadhwa, B

Wagh, D

Wakiyama, Y

Wan, X
754

1142

1004

733

251

632

423

1004

238

632

238

954

226

98

246

878

246

871

788

805

775

685

962

251

264

429, 1133

1070

582,611

$52,655,888,1112$

987

691

1133

251

937

130

259

152

859

1065

1122

212

856

1097

1078, 1150

212

308

2, 655, 888, 1112

190, 219

Wang, B-Y

313

Wang, $\mathrm{H}$

297, 832

Wang, J-D

1026

Wang, J-D $\quad 187,190,216,219,297$

Wang, J

Wang, $\mathrm{K}$

Wang, L

Wang, M-H

763

915

Wang, N

158,987

Wang, Q

801,1138

710

Wang, $\mathrm{R}$

443,715

743

923

Wang, W

Wang, $\mathrm{X}$

90, 923, 970

Wang, X-d

285

Wang, X-J

187,1026

Wang, X-W

801

1029

853

Wang, YS

788,819

Wang, Z

705

Watanabe, T 52, 542, 655, 888, 1146

Watanabe, Y

$331,395,675$

Watanapokasin, $\mathrm{R}$

109

Watts, RE

308

Wei, X-Y

1047

Weisblum, B

918

Wellington, $\mathrm{E}$

73

Wewengkang, DS 967

Wichner, D

448

Wo, J

90

Wright, GD

366

$\mathrm{Wu}, \mathrm{A}-\mathrm{a}$

1029

$\mathrm{Wu}, \mathrm{J}$

1009

$\mathrm{Wu}, \mathrm{L}$

158

$\mathrm{Wu}, \mathrm{S}$

90

Xiang, W-S 187, 216, 297, 1026

Xiao, YS

853

Xie, D

90

Xie, Y

970

Xiong, W

915

$\mathrm{Xu}, \mathrm{G}$

743

$\mathrm{Xu}, \mathrm{J}$

733

$\mathrm{Xu}, \mathrm{L}$

671

$\mathrm{Xu}, \mathrm{Q}$

1029

$\mathrm{Xu}, \mathrm{X}$

923

$\mathrm{Xu}, \mathrm{Z}$

414

Yagi, A 
Yamamura, $\mathrm{H}$

Yamasaki, M

Yamasaki, Y

Yamashita, K

Yamazaki, H

Yan, B-F

Yan, $\mathrm{H}$

Yan, $\mathrm{K}$

Yang, B

Yang, C-Y

Yang, CL

Yang, D

Yang, $\mathrm{H}$

Yang, J-Y

Yang, L

Yang, S-s

Yang, Y-B

Yao, J-N

Yao, Q-F

Ye, Y

Yedukondalu, N

Yeh, PJ

Yin, $\mathrm{Y}$

Yixizhuoma, 601

Yokoigawa, J

Yonejima, K
995 Yoon, E-J

582 Yoshida, I

691 Yoshida, J

699 Yoshida, M

331, 967 Yoshida, T

1047 Yoshikawa, K

301 Yoshikawa, T

187 Yoshimura, S

788 You, X

823, 1047 Young, K

$853 \mathrm{Yu}, \mathrm{B}$

200, 414, $710 \quad$ Yu, E

832 Yu, L

147 Yu, M

853 Yurchenko, AN

285 Yurchenko, EA

313, 832 Yuzawa, S

1104

763

166

212

1033

193

Zahraie, Z

Zha, D-M

Zhai, J

Zhang, B-H

Zhang, C

Zhang, D-J

929

708

Zhang, F
400 Zhang, J

264 Zhang, J-C

1133 Zhang, L

1085 Zhang, M

655 Zhang, Q-B

41 Zhang, S-y

695, 979 Zhang, X

45 Zhang, X-E

158 Zhang, X-Y

671 Zhang, XY

1065, 1122 Zhang, Y

828 Zhao, J

105 Zhao, L-X

801 Zhao, Y

856 Zhao, Z-Z

856 Zheng, C-J

378 Zheng, Z

845

147

147,853

823

733

970

Zhang, H 187, 190, 216, 219, 297
Zhou, $\mathrm{H}$

Zhou, WY

Zhou, $\mathrm{X}$

Zhu, C

$187,216,297,715,915$

313

715,1029

853

193, 801

190, 297

158

285

$715,763,970$

301

105, 193, 819, 1138

105

$200,313,314$

1026

1104

823

158

313

301

788

443

174

Zhu, T

Zhu, X

Zou, Z-M

200, 414, 743, 819

801 